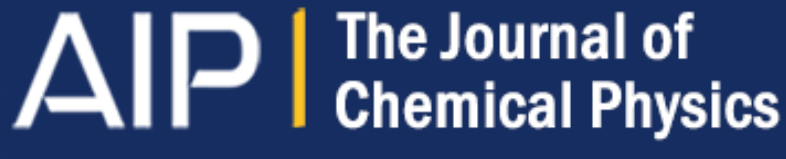

\section{Hydrogen molecule ion in superintense laser fields}

C. A. S. Lima and L. C. M. Miranda

Citation: The Journal of Chemical Physics 78, 6102 (1983); doi: 10.1063/1.444571

View online: $\mathrm{http} / / / \mathrm{dx}$.doi.org/10.1063/1.444571

View Table of Contents: http://scitation.aip.org/content/aip/journal/jcp/78/10?ver=pdfcov

Published by the AIP Publishing

\section{Articles you may be interested in}

Dimensional scaling treatment with relativistic corrections for stable multiply charged atomic ions in highfrequency super-intense laser fields

J. Chem. Phys. 136, 034114 (2012); 10.1063/1.3673317

Superintense fields from multiple ultrashort laser pulses retroreflected in circular geometry J. Appl. Phys. 107, 043110 (2010); 10.1063/1.3296128

Dimensional scaling treatment of stability of simple diatomic molecules induced by superintense, high-frequency laser fields

J. Chem. Phys. 129, 214110 (2008); 10.1063/1.3027451

New stable multiply charged negative atomic ions in linearly polarized superintense laser fields

J. Chem. Phys. 124, 201108 (2006); 10.1063/1.2207619

X-ray pulse train generation by long-lived atoms and ions in superintense laser field AIP Conf. Proc. 611, 21 (2002); 10.1063/1.1470285

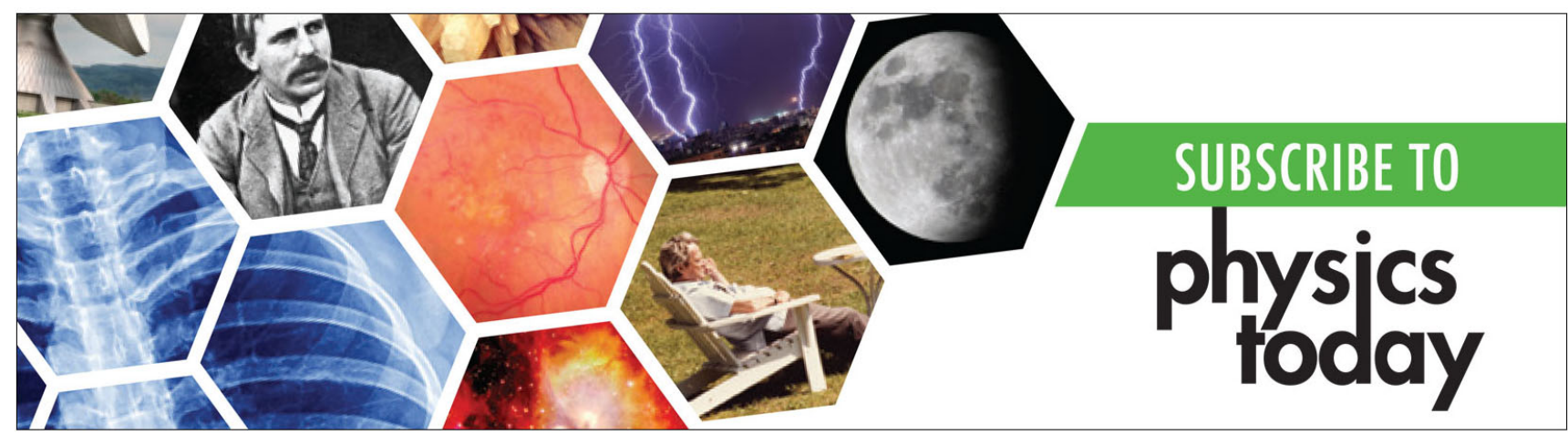




\title{
Hydrogen molecule ion in superintense laser fields
}

\author{
C. A. S. Lima \\ Instituto de Fisica, Universidade Estadual de Campinas, 13100 Campinas, SP, Brazil
}

L. C. M. Miranda

Divisão de Estudos Avancados, Centro Técnico Aeroespacial, 12200 S. J. Campos, SP, Brazil (Received 30 March 1982; accepted 3 February 1983)

The structure of $\mathrm{H}_{2}^{+}$under an intense laser field is discussed. It is shown that the dissociation energy decreases on increasing the laser field strength and a discussion on the validity criteria of the theory is also given.

In recent years, considerable effort has been devoted to the description of atoms in the presence of intense nonresonant laser fields. ${ }^{1-7}$ In contrast, in the case of laser-molecule interaction the attention has almost invariably been focused on the interaction of intense resonant laser radiation aiming, in particular, high vibrational selective excitation of molecules. ${ }^{8-10}$ In the present work, we report on the structure of molecules in the presence of nonresonant super-intense laser fields paying particular attention to the changes induced by such radiation fields on the confinement and stabilization of molecules. The laser beam is treated as a classical plane electromagnetic wave of frequency $\omega$, in the dipole approximation, and only its interaction with the electrons is taken into account. Furthermore, we shall consider as an example for our model calculation the simplest molecule, namely, the hydrogen molecule ion. Hence, neglecting the interaction of the laser field with the nuclei $A$ and $B$, and denoting by $r_{A}$ and $r_{B}$ the distances between the electron and the nuclei $A$ and $B$, respectively, the molecular Hamiltonian can be written as

$$
\begin{aligned}
& H=T_{R}+H_{0}, \\
& H_{0}=\frac{1}{2 m}\left[\hat{p}+\frac{e}{c} \mathrm{~A}(t)\right]^{2}-\frac{e^{2}}{r_{\mathrm{A}}}-\frac{e^{2}}{r_{\mathrm{B}}}+\frac{e^{2}}{R},
\end{aligned}
$$

where $A(t)=A(\hat{x} \cos \omega t+\hat{y} \sin \omega t)$ is the vector potential for a circularly laser beam propagating in the $z$ direction, $T_{R}$ is the kinetic energy operator of the nuclei, and $R$ is the internuclear separation. In Eq. (1), $H_{0}$ describes the electron motion for fixed internuclear distance $R$, and its ground-state energy $E(R)$ is, within the adiabatic approximation, the potential energy for the nuclei motion.

In the following, we discuss in detail the electronic ground state. Accordingly, the problem of finding the binding potential of $\mathrm{H}_{2}^{*}$, within the adiabatic approximation, is then reduced to the solution to the ground state of the Schrödinger equation for $H_{0}$, namely,

$$
i \hbar \dot{\psi}=H_{0} \psi \text {. }
$$

To this end, we begin by performing a canonical transformation $^{3,6,7}$ in Eq. (2), i.e.,

$$
\psi=\exp [i \otimes(t) \cdot \hat{p} / \hbar] \exp [i \eta(t) / \hbar] \phi,
$$

where

$$
\delta(t)=\frac{e}{m c} \int^{t} d t^{\prime} A\left(t^{\prime}\right), \quad \eta(t)=\frac{e^{2}}{2 m c^{2}} \int^{t} d t^{\prime} \mathrm{A}^{2}\left(t^{\prime}\right),
$$

such that in this new representation the Schrödinger equation for the electron motion becomes

$$
i \hbar \dot{\phi}=\left\{\frac{\hat{p}^{2}}{2 m}-\frac{e^{2}}{\left|\mathbf{r}_{\mathrm{A}}-\delta\right|}-\frac{e^{2}}{\left|\mathbf{r}_{\mathrm{A}}-\delta\right|}+\frac{e^{2}}{R}\right\} \phi .
$$

Equation (5) tells us that the effect of an intense laser field on the molecular structure may be alternatively described by the Schrödinger equation in which the nuclei appear to be oscillating with the laser frequency and amplitude $|\delta(t)|=a=e A / m c \omega$. Furthermore, this laser-dressed distortion of the Coulomb points in the direction of a weakening of the electron binding to the nucleus on increasing the laser field strength. We also note that Eq. (5) is not separable and therefore has no exact analytical solutions. This means that when describing the distortion of the molecular states by a laser field, there should always exist a limit on the laser intensity such that one can still talk about the molecule as being in a stationary laser-dressed state. In other words, for each "dressing" laser there is a corresponding range of laser intensities in which Eq. (5) can be analytically solved (or, at least, some suitable approximation can be found) thereby leading to the appearance of the laser-dressed states. As we shall see later on, this range of laser intensity is essentially determined by the fact that, as one increases the laser field strength, the dissociation energy as well as the transition energy between the lowest bonding and antibonding states of $\mathrm{H}_{2}^{+}$decreases. This means that, on increasing the laser field, one eventually reaches a point in which the laser photon energy equals the first electronic transition so that we can no longer assume the molecule to be in the stationary ground state.

Following the same reasoning as in Refs. 7, 11, and 12, the laser-dressed Coulomb potentials in Eq. (5) can be written as

$$
\begin{aligned}
-\frac{e^{2}}{\left|\mathbf{r}_{i}-\delta(t)\right|} & =-\frac{e^{2}}{\left(r_{i}^{2}+a^{2}\right)^{1 / 2}}\left[1-\frac{2 \mathbf{r}_{i} \cdot \delta(t)}{r_{i}^{2}+c^{2}}\right]^{-1 / 2} \\
& =-\frac{e^{2}}{\left(r_{i}^{2}+a^{2}\right)^{1 / 2}}\left\{1+\frac{r_{i} \cdot \delta(t)}{r_{i}^{2}+a^{2}}+\ldots\right\},
\end{aligned}
$$

where $a=e A / m c \omega$ and $i=A, B$. Noticing that $\left(r_{i} \cdot 8(t) /\right.$ $\left.r_{i}^{2}+a^{2}\right)^{n}$ will never exceed $(1 / 2)^{n}$ we can safely assume that the binding potential is adequately described, in 
lowest order, by the first term of expansion (6), namely,

$$
-\frac{e^{2}}{\left|\mathbf{r}_{i}-\delta(t)\right|} \simeq-\frac{e^{2}}{\left(r_{i}^{2}+a^{2}\right)^{1 / 2}}
$$

Making this approximation in Eq. (5), the electron motion is then described, in lowest order, by

$i \hbar \dot{\phi}=\left\{\frac{p^{2}}{2 m}-\frac{e^{2}}{\left(r_{\mathrm{A}}^{2}+a^{2}\right)^{1 / 2}}-\frac{e^{2}}{\left(r_{\mathrm{B}}^{2}+a^{2}\right)^{1 / 2}}+\frac{e^{2}}{R}\right\} \phi$.

To solve Eq. (8) for the electronic ground state we resort to the variational method. Accordingly, we write the wave function in the form of a linear combination of the wave functions of the electron moving independently in the field of the laser-dressed Coulomb potential of the nuclei $A$ and $B$, namely,

$$
\phi=c_{\boldsymbol{i}} \phi_{\mathbf{A}}+c_{2} \phi_{\mathbf{B}} \text {. }
$$

However, instead of using for $\phi_{A}$ and $\phi_{B}$ the groundstate functions of the laser-dressed hydrogen atom as given in Ref. 7, we write $\phi_{\mathbf{A}, \mathbf{B}}$ as

$$
\phi_{\mathbf{A}, \mathbf{B}}=\left(\gamma^{3} / \pi\right)^{1 / 2} \exp \left(-\gamma r_{\mathbf{A}, \mathbf{B}}\right) \text {, }
$$

where $\gamma$ is a variational parameter to be determined from the requirement that the energy should be minimum for a fixed distance between the nuclei.

Substituting Eqs. (9) and (10) into Eq. (8), and performing the standard calculations ${ }^{13}$ to determine the parameters $c_{1}, c_{2}$, and $\gamma$, the electronic wave function and energy for the $1 s \sigma$ state can be written as

$$
\begin{aligned}
& \phi=[2(1+S)]^{-1 / 2}\left(\phi_{\mathbf{A}}+\phi_{B}\right), \\
& E=\frac{e^{2}}{2 a_{0}}\left\{\frac{1}{x}+\frac{\left(\rho^{2} / x^{2}\right)(1+I)-\left(\rho^{3} / x\right) N}{(1+S)}\right\},
\end{aligned}
$$

where $x=R / a_{0}, \rho=\gamma R$, and

$S=\left(1+\rho+\frac{\rho^{2}}{3}\right) e^{-\rho} ; \quad I=\left(1+\rho-\frac{\rho^{2}}{3}\right) e^{-\rho}$,

$N=\int_{1}^{\infty} d \mu e^{-\rho \mu} \int_{-1}^{+1} d \nu \frac{2\left(\mu^{2}-\nu^{2}\right)[\cosh (\rho \nu)+1]}{\left[(\mu+\nu)^{2}+(2 \lambda / x)^{2}\right]^{1 / 2}}$,

in which the parameter $\rho$ (i.e., $\gamma$ ) is determined by re-

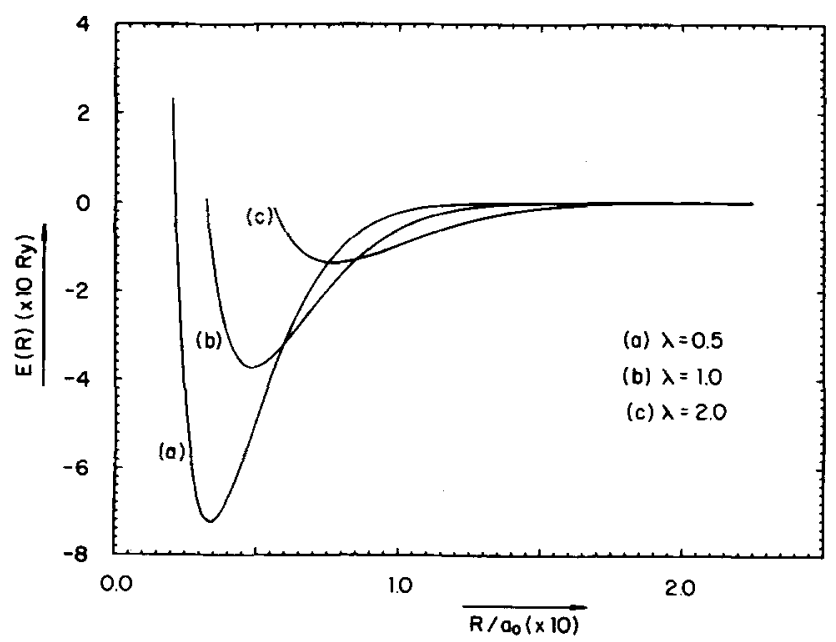

FIG. 1. Ground -state potential energy $E(R)$ (in Ry) vs the internuclear separation (in a.u.) for $\mathrm{H}_{2}^{*}$ at several laser field strength settings.

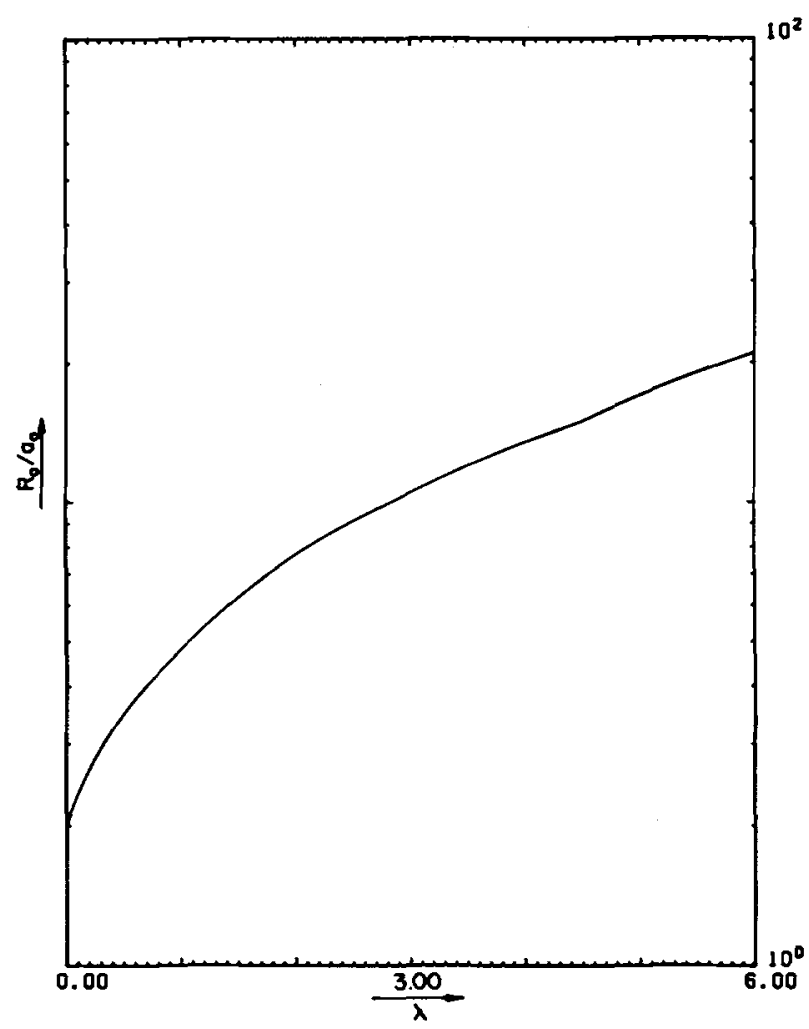

FIG. 2. Equilibrium internuclear distance $R_{0}$ (in a. u.) of $\mathrm{H}_{2}^{+}$ as a function of the laser field strength.

quiring that $\partial E / \partial \rho=0$. The integrals in Eqs. (13) have no simple analytical expressions and were evaluated numerically for several values of the laser field strength and internuclear distances. In our calculations we have introduced the dimensionless parameter $\lambda=a / a_{0}\left(a_{0}\right.$ $=$ Bohr radius) as a measure of the laser field strength. The parameter $\lambda$ is related to the laser frequency $\omega$ (in $\mathrm{s}^{-1}$ ) and intensity $I$ (in W/cm ${ }^{2}$ ) by $\lambda=6.44 \times 10^{24} \omega^{-2} I^{1 / 2}$. In Fig. 1, we show the potential energy $E(R)$ as a function of the distance between the nuclei taking the zero of energy at the atomic ground state. In Table I we summarize the values of the equilibrium internuclear distance $R_{0}$ and the dissociation energy $D$ for several laser power settings. The variations of the equilibrium internuclear distance and of the dissociation energy as a function of the laser field are presented in Figs. 2

TABLE I. Equilibrium internuclear distance $R_{0}$ and dissociation energy $D$ of $\mathrm{H}_{2}^{+}$as a function of the laser field strength parameter $\lambda$. The parameter $\lambda$ is related to the laser intensity $I$ (in W/c m ${ }^{2}$ ) by $\lambda=6.44 \times 10^{24} \omega^{-2} I^{1 / 2}$.

\begin{tabular}{lcc}
\hline$\lambda$ & $R_{0}$ (a.u.) & $D(\mathrm{Ry})$ \\
\hline 0 & 2 & 0.1710 \\
0.5 & 3.5 & 0.0713 \\
1 & 4.8 & 0.0365 \\
2 & 7.7 & 0.0128 \\
3 & 10.5 & 0.0049 \\
4 & 13.5 & 0.0023 \\
4.5 & 15.5 & 0.0014 \\
5.0 & 17 & 0.0008 \\
5.5 & 19 & 0.0003 \\
\hline \hline
\end{tabular}




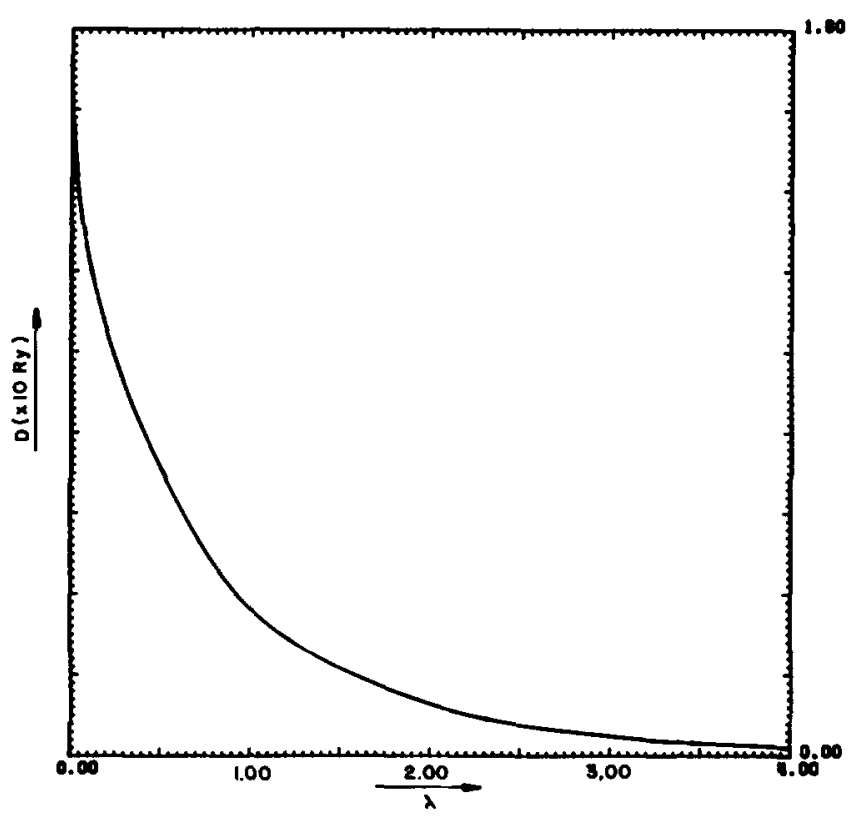

FIG. 3. Dissociation energy $D$ (in Ry) of $\mathrm{H}_{2}^{+}$as a function of the laser field strength.

and 3 , respectively. It follows from these plots that, as the laser field increases, $R_{0}$ increases at the same time that $D$ decreases thereby confirming our expectation that the effect of an intense laser field is to weaken the molecule confinement and stability. This weakening effect of the laser field on the molecule structure is particularly evident from Fig。 1 , where it is shown that the potential curves become flater and shallower on increasing the laser intensity. This means that on increasing the laser field the molecule becomes highly anharmonic at the same time that its dissociation via low energy (or thermal fluctuations) is greatly enhanced. In fact, looking at Fig. 3 , one notices that for $\lambda=\lambda_{\mathrm{th}}=3.75$ the dissociation energy is already equal to the thermal energy at room temperature $\left(\epsilon_{\text {th }}=0.0029\right.$ Ry). At the same time, it follows from Fig. 2 that at this value of $\lambda(=3.75), R_{0}$ begins to grow exponentially. Thus, combining all these facts, one is then led to the conclusion that, under intense laser illumination, the dissociation of diatomic molecules might be more likely to take place via low-energy (or thermal) processes rather than via the stepwise multiphoton vibrational excitation.

To conclude, a final word regarding the range of applicability for our model to be valid is in order. The main assumption used here is the dipole approximation for describing the laser field. As already discussed in Refs. 7, 11, and 12 the use of the dipole approximation sets an upper limit intensity $I_{u}$ for our method to be valid, namely,

$$
I \ll I_{u},
$$

where

$$
I_{u}=\frac{m^{2} c^{3} \omega^{2}}{4 \pi e^{2}}=0.77 \times 10^{-12} \omega^{2}\left(\mathrm{~W} / \mathrm{cm}^{2}\right)
$$

This means that for a $\mathrm{CO}_{2} \operatorname{laser}\left(\omega=1.78 \times 10^{14} \mathrm{~s}^{-1}\right)$ our model is valid for intensities up to roughly $2.44 \times 10^{16}$ $\mathrm{W} / \mathrm{cm}^{2}\left(\lambda \sim 10^{4}\right)$, whereas for a Nd: glass laser $(\omega=1.78$ $\left.\times 10^{15} \mathrm{~s}^{-1}\right)$ the upper intensity is of the order of $10^{18}$ $\mathrm{W} / \mathrm{cm}^{2}$. Actually, this upper intensity is, by all means, only of academic interest since much before the laser intensity reaches these values the molecule may be completely dissociated. Here the point to be noted is that both the dissociation energy as well as the energy separation $\Delta \epsilon$ for the $1 s \sigma-2 p \sigma$ states transition of $\mathrm{H}_{2}$ decrease with increasing laser intensity. The transition energy $\Delta \epsilon$, at the equilibrium internuclear separation $R_{0}$, can be evaluated by calculating the $2 p \sigma$ state potential curve for the dressed Hamiltonian [cf., Eq. (8)] following the same procedures as for the bonding state. In Fig. 4 we plot the dependence of $\Delta \epsilon$ on the laser field strength. It follows from Fig. 4 that, on increasing the laser intensity one eventually reaches a point $\lambda^{*}$ where photodissociation due to the dressing laser photon itself takes place. For example, let us consider the case of $\mathrm{H}_{2}^{+}$irradiated by a $\mathrm{CO}_{2}$ laser. The first electronic transition, due to the $\mathrm{CO}_{2}$ laser photon itself is expected to occur at $\lambda^{*}=5.65$ or $I \simeq 7.7 \times 10^{8}$ $\mathrm{W} / \mathrm{cm}^{2}$. In contrast, in the case of a $\mathrm{Nd}$ : glass laser the photodissociation by the laser photon itself occurs at $\lambda^{*}=1.9$ (or $\left.I \simeq 8.7 \times 10^{11} \mathrm{~W} / \mathrm{cm}^{2}\right)$. This means that for $\mathrm{H}_{2}^{+}$irradiated by a $\mathrm{CO}_{2}$ laser, and neglecting the thermal fluctuations, we can assume it to be in the stable laser-dressed ground state only for $\lambda$ up to $\lambda^{*}$ $=5.65$.

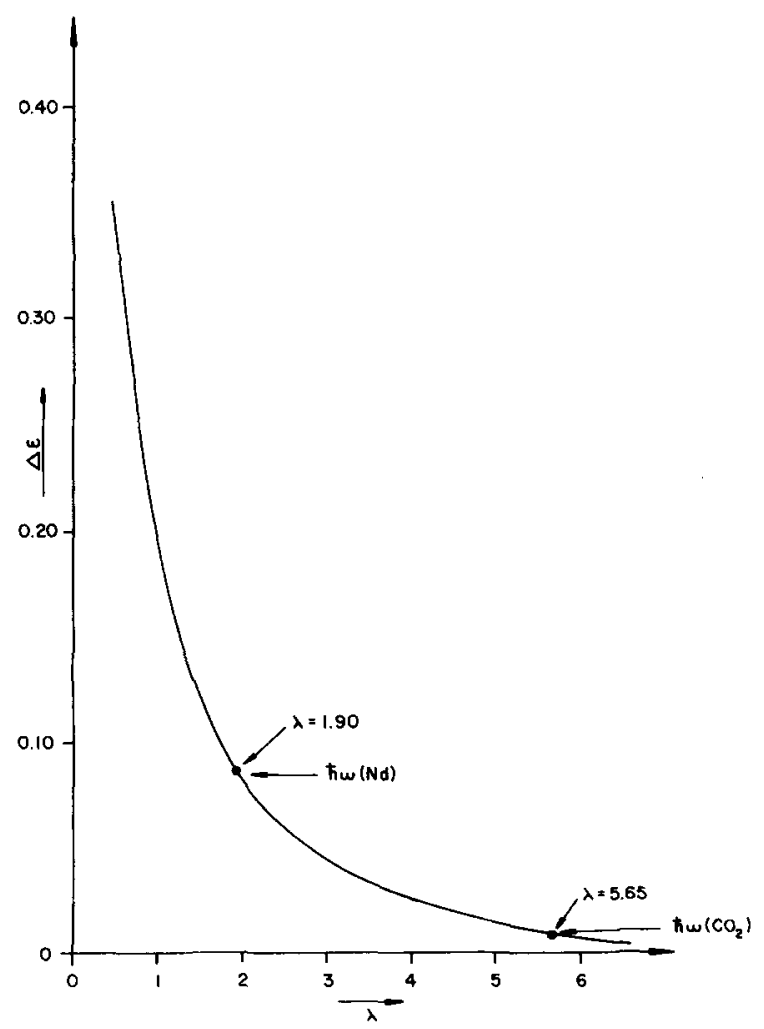

FIG. 4. Energy separation $\Delta \varepsilon$ (in Ry) for the $1 s \sigma-2 p \sigma$ transition of $\mathrm{H}_{2}^{+}$, at the equilibrium internuclear distance $R_{0}$, as a function of the laser field strength. The arrows indicate the points at which photodissociation due to some well known laser photons occurs. 
To sum up, one may say that, upon increasing the laser intensity, $\mathrm{H}_{2}^{*}$ acquires new stable laser-dressed bonding such that its dissociation energy decreases with increasing laser field. Its identification in this state is only possible in a range of laser intensities such that neither photodissociation or dissociation due to thermal fluctuations occurs, i.e., this range of $\lambda$ is essentially determined by the smallest between $\lambda_{\text {th }}$ and $\lambda^{*}$. Furthermore, this range of laser intensity is ultimately. dictated by the dressing laser frequency. For example, if one dresses $\mathrm{H}_{2}^{+}$by a relatively low frequency laser such as a $\mathrm{CO}_{2}$ laser, the actual range of laser intensities is up to $\lambda=3.75$, since at this value of $\lambda$ the thermal fluctuations are sufficient to dissociate it. In contrast, if one uses higher frequency lasers such as a $\mathrm{Nd}$ : glass laser, the range of intensity is up to $\lambda=1.9$ since at this point the molecule undergoes photodissociation, as indicated in Fig. 4. It also follows from this discussion that the use of low frequency radiation fields strongly favors the dissociation of the molecule. The reason this is that $\lambda$ varies as $\omega^{-2}$ (i.e., $\lambda=e E_{0} / m \omega^{2} a_{0}$ ) so that very large values of $\lambda$ (such that $\lambda \gg \lambda_{\text {th }}$ or $\lambda^{*}$ ) are attainable in the low frequency range. Even though, there is no detailed experimental data on the dissociation of $\mathrm{H}_{2}^{+}$under intense radiation fields available to us, we believe the above conclusions are in agreement with a few experimental facts. One of these is the $\mathrm{rf}$ or the microwave preionization mechanism used in gaseous lasers, such as a $\mathrm{CO}_{2}$ laser. What one does here is to introduce a rf finger at very small power levels in the laser medium; the gas gets ionized almost immediately even under low pressure conditions, as expected from the above discussion.

${ }^{1}$ L. V. Keldysh, Sov. Phys. JETP 20, 1307 (1965).

${ }^{2}$ W. C. Henneberger, Phys. Rev. Lett. 21, 838 (1968).

${ }^{3}$ H. R. Reiss, Phys. Rev. D 4, 3533 (1971).

${ }^{4} \mathrm{M} . \mathrm{H}$. Mittleman, Phys, Lett. A 47, 55 (1974).

${ }^{5}$ M. Jain and N. Tzoar, Phys. Rev, A 16, 147 (1977).

${ }^{6}$ H. S. Brandi, B. Koiller, H. G. P. Lins de Barros, L. C. M. Miranda, and J. J. Castro, Phys. Rev. A 17, 1900 (1978). ${ }^{7}$ C. A. S. Lima and L. C. M. Miranda, Phys. Rev. A 23, 3335 (1981).

${ }^{8}$ N. Bloembergen, Opt. Commun. 15, 416 (1975).

${ }^{9}$ E. M. Belenov, S. Yu. Cuskov, and U. A. Isakov, Sov. J. Quant. Electron, 4, 1135 (1975).

${ }^{10}$ A. Lau, Phys. Rev. A 18, $172(1978)$, and references therein.

${ }^{11}$ N. S. Almeida and L. C. M. Miranda, Opt. Commun. 42, 241 (1982).

${ }^{12}$ T, C. Landgraff, J. R. Leite, N. S. Almeida, C. A. S. Lima, and L. C. M. Miranda, Phys. Lett. A 92, 131 (1982).

${ }^{13}$ A. S. Davydov, Quantum Mechanics (Pergamon, Oxford, 1965), p. 484. 